\title{
Analysis of a New Quadratic 3D Chaotic Attractor
}

\author{
Shahed Vahedi and Mohd Salmi Md Noorani \\ School of Mathematical Sciences, Universiti Kebangsaan Malaysia, 43600 Bangi, Selangor, Malaysia \\ Correspondence should be addressed to Shahed Vahedi; shv.math@gmail.com
}

Received 3 February 2013; Revised 10 April 2013; Accepted 28 April 2013

Academic Editor: Antonio Suárez

Copyright (C) 2013 S. Vahedi and M. S. M. Noorani. This is an open access article distributed under the Creative Commons Attribution License, which permits unrestricted use, distribution, and reproduction in any medium, provided the original work is properly cited.

\begin{abstract}
A new three-dimensional chaotic system is introduced. Basic properties of this system show that its corresponding attractor is topologically different from some well-known systems. Next, detailed information on dynamic of this system is obtained numerically by means of Lyapunov exponents spectrum, bifurcation diagrams, and 0-1 chaos indicator test. We finally prove existence of this chaotic attractor theoretically using Shil'nikov theorem and undetermined coefficient method.
\end{abstract}

\section{Introduction}

The current surge towards the study on dynamical systems and $3 \mathrm{D}$ chaotic attractors started by the remarkable discovery of Lorenz in 1963 [1]. While he was studying atmospheric convections using Saltzman equations, he finally came up with a new system of differential equations (1), which is known after his name. His further investigations on this new system showed that for specific values of parameters it has a new type of attractor, namely, chaotic attractor as follows:

$$
\begin{gathered}
\dot{x}=a(y-x), \\
\dot{y}=c x-y-x z, \\
\dot{z}=x y-b z .
\end{gathered}
$$

During the past decades, enormous amount of researches have been done on this system which have revealed characteristics and features of it [2].

Besides the researches on Lorenz, many other system of equations have been introduced and analysed since then, like Chen, Rössler, Chua, Lü, Qi, and many others. For instance, a new chaotic system was reported with no equilibria which is based on Sprott D system [3]. In another research, a chaotic system with only one stable equilibrium was introduced which typically is not anticipated to show such a behaviour [4]. And recently a technique to construct a chaotic system with an arbitrary number of equilibria has been also introduced [5]. Since the idea of the proposed system has come from Chen and Qi systems, we describe these two systems briefly.

The Chen system (2) is constructed using a state feedback in the second equation of Lorenz, and he showed that this system is chaotic at $a=35, b=3$, and $c=28$ as follows:

$$
\begin{gathered}
\dot{x}=a(y-x), \\
\dot{y}=(c-a) x+c y-x z, \\
\dot{z}=x y-b z .
\end{gathered}
$$

Later on, by eliminating the first term in the second equation, Chen and Lü proposed the Lü system, which is infact the transition between Lü and Chen [6].

In 2005, Qi and his colleagues added a cross-product nonlinear term to the first equation of Lorenz and introduced a new system (3) that was topologically different from Lorenz, Chen, Rössler, and even Lorenz system family [7] as follows:

$$
\begin{gathered}
\dot{x}=a(y-x)+y z, \\
\dot{y}=c x-y-x z, \\
\dot{z}=x y-b z .
\end{gathered}
$$


Comparing (2) and (3) with Lorenz shows that how small modifications on Lorenz could lead to a system with new characteristic features and in some cases even topologically different attractor. The system we are going to introduce and study here is

$$
\begin{gathered}
\dot{x}=a(y-x)+y z, \\
\dot{y}=(c-a) x+c y-x z, \\
\dot{z}=x y-b z .
\end{gathered}
$$

It has a chaotic attractor at $a=15, b=8 / 3$, and $c=10$ which is topologically different from Lorenz, Chen, and even Rössler to be shown shortly.

This paper is divided into five parts. Section 2 deals with studying the basic properties of system (4). After that in Section 3, more detailed investigation will be done on dynamic of this system using Lyapunov exponents spectrum, bifurcation diagrams, and $0-1$ test which reveals the behaviour of this system for various sets of parameters, and how it evolves from one state to the other. Then in Section 4 we will theo-retically prove the existence of chaos in this system using Shil'nikov theorem and undetermined coefficient method [8]. We conclude this paper in Section 5.

\section{Basic Properties}

In this section, we start with equilibrium points of the system and check their stability at initial values of parameters $a, b$, and $c$. Putting equations of the system equal to zero gives the equilibrium points

$$
\begin{gathered}
v_{1}=(0,0,0), \\
v_{2,3}=\left( \pm \frac{a \sqrt{2} \sqrt{c d b a}}{2 c(a-(d a / 2 c))}, \pm \frac{\sqrt{2} \sqrt{c d b a}}{2 c}, \frac{a^{2} d}{2 c(a-(d a / 2 c))}\right), \\
v_{4,5}=\left( \pm \frac{a \sqrt{-2 c e b a}}{2 c(a+(e a / 2 c))}, \pm \frac{\sqrt{-2 c e b a}}{2 c},-\frac{a^{2} e}{2 c(a+(e a / 2 c))}\right),
\end{gathered}
$$

where

$$
\begin{aligned}
& d=3 c+\sqrt{c^{2}+4 c a} \\
& e=-3 c+\sqrt{c^{2}+4 c a} .
\end{aligned}
$$

Therefore, at the initial values of parameters $a, b$, and $c$, this system has five equilibrium points in contrast to Lorenz, Chen, and Rössler which have three, three, and two equilibriums, respectively. It implies topological distinction between system (4) and these systems.

In order to check their stability we derive Jacobian matrix of the system

$$
D f(x, y, z)=\left(\begin{array}{ccc}
-a & a+z & y \\
c-a-z & c & -x \\
y & x & -b
\end{array}\right) .
$$

Substituting the origin in this matrix we get the characteristic equation

$$
\left(\lambda^{2}+(-c+a) \lambda+a(-2 c+a)\right)(\lambda+b)=0
$$

which has three eigenvalues $\lambda_{1}=6.5139, \lambda_{2}=-2.6667$, and $\lambda_{3}=-11.5139$. Since all the eigenvalues are real, HartmanGrobman theorem implies that origin is a saddle point which is not Lyapunov stable according to the Lyapunov theorem of stability.

The eigenvalues of the Jacobian at $v_{2}$ and $v_{3}$ are $\lambda_{1}=$ -19.5735 and $\lambda_{2,3}=5.9534 \pm 11.4898 i$; notice that these two points are symmetrically located around $z$ axis. None of these numbers have real part zero, and because $\lambda_{2,3}$ are complex, $v_{2}$ is an unstable saddle-focus point. Exactly the same results holds for $v_{3}$.

At $v_{4}$ and $v_{5}$ the eigenvalues are $\lambda_{1}=-10.3231$ and $\lambda_{2,3}=$ $1.3282 \pm 6.5092 i$. Similar to $v_{2,3}$, it shows that this system has a saddle focus at $v_{4}$ and $v_{5}$ that is not Lyapunov stable.

A large class of chaotic systems are dissipative dynamical systems which satisfy the condition

$$
\nabla \cdot V=\frac{\partial \dot{x}}{\partial x}+\frac{\partial \dot{y}}{\partial y}+\frac{\partial \dot{z}}{\partial z}<0
$$

In the case of system (4) we have $\nabla \cdot V=c-(a+b)=10-$ $(15+8 / 3)<0$; therefore dissipativity condition holds on this system. Moreover

$$
\frac{d V}{d t}=e^{c-(a+b)}=0.0004682 .
$$

It implies that the volume of the attractor decreases by a factor of 0.0004682 and at each particular time $t$ the volume is

$$
V(t)=V_{0} e^{(c-(a+b)) t} .
$$

The key characteristic of a chaotic system is its sensitive dependency on initial conditions; which is associated with having positive Lyapunov exponents. Using the MATLAB program at [9], which is based on the algorithm introduced in [10], the Lyapunov exponents of the system are $0.766 \pm 0.005$, $0 \pm 0.0004$, and $-8.435 \pm 0.005$. In addition, exponentiating these numbers indicates that nearby trajectories diverge from each other by a factor of 2.151 .

Having the Lyapunov exponents, Kaplan-Yorke (Lyapunov) dimension [11] of the attractor is 2.0908. On the other hand, using correlation and box-counting methods, fractal dimensions of the attractor are 1.9763 and 0.9405 , respectively, which indicate its fractal structure. Figure 1 shows the attractor and its projections on different planes.

\section{Computational Analysis}

In this section, we employ extensive computations to have a broader picture of the dynamic of this system in different regions of parameters' values. Our focus is on the parameter $a$, but we do show the results regarding $b$ and $c$ in the figures. 


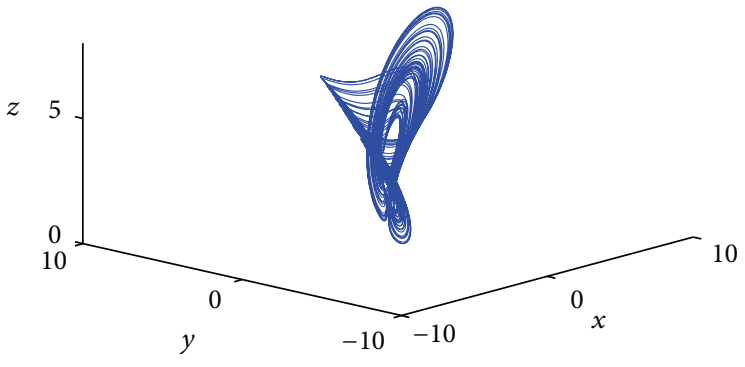

(a)

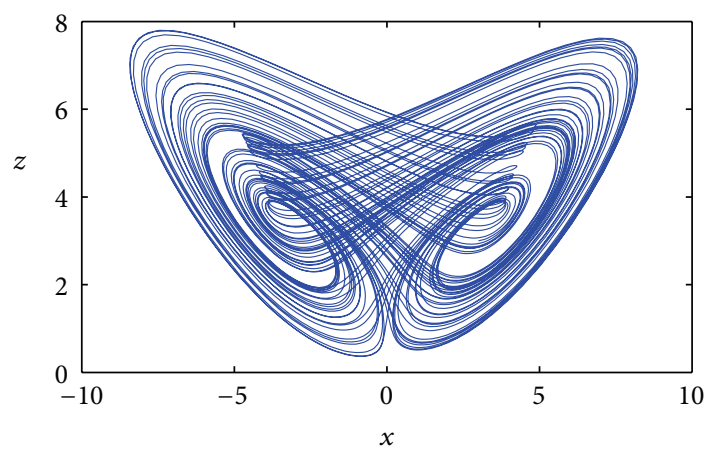

(c)

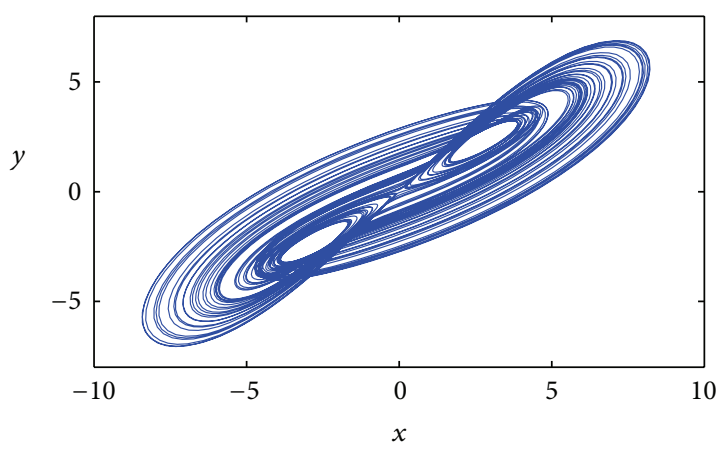

(b)

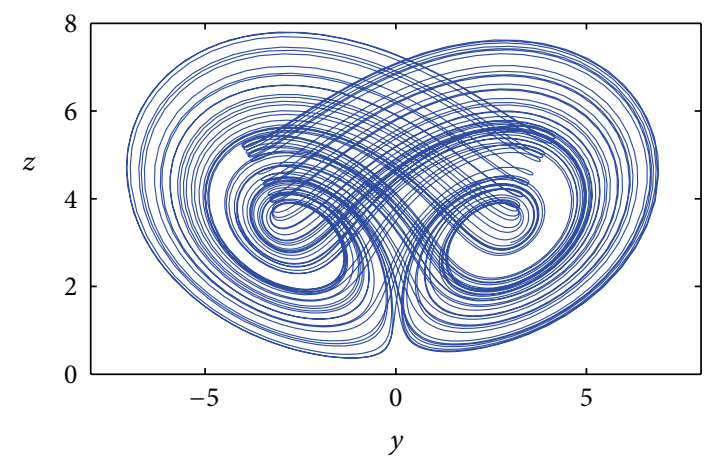

(d)

FIGURE 1: 3D view of the attractor and its various projections.

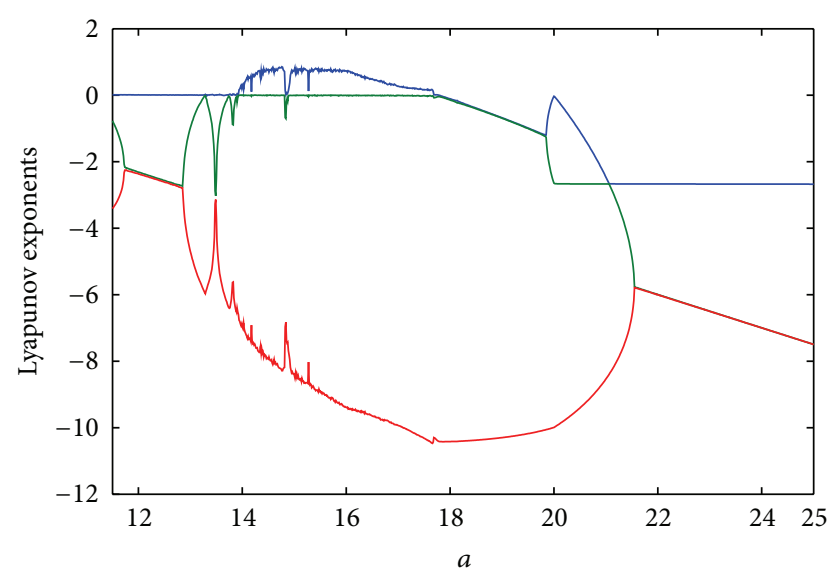

FIGURE 2: Lyapunov exponents spectrum of the system with respect to $a$.

At different set of parameters' values, behaviour of a system could be either chaotic, periodic, or convergence to one of the equilibria. So to have a good understanding of its dynamics, we need to identify the regions whereby the system is in either of these states. Here we use Lyapunov exponents spectrum, bifurcation diagrams, and $0-1$ chaos indicator test [12] to obtain this information.

Figure 2 shows the Lyapunov exponents spectrum with respect to parameter $a \in[11.5,25]$. As we see, at the beginning the system is in periodic state, and trajectories converge to a limit cycle in the state space. At $a=13.94$, chaotic

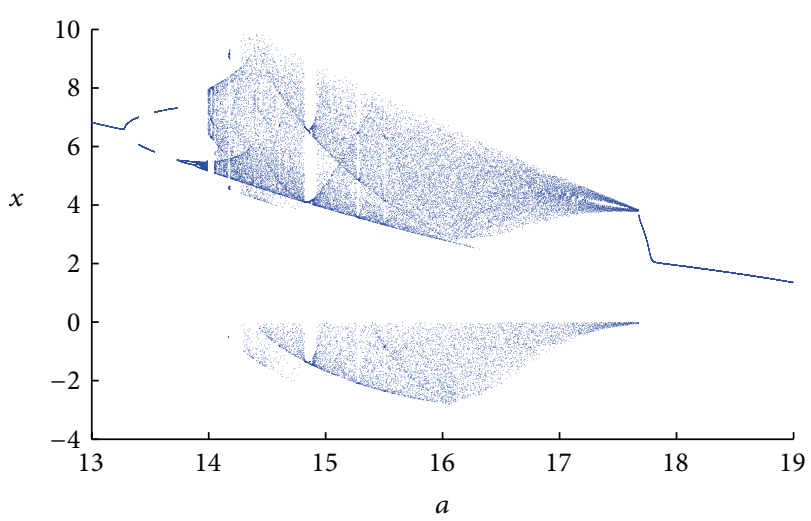

Figure 3: Bifurcation diagram of the system with respect to $a$.

state emerges, which means that the system becomes sensitive to its initial condition. It continues up to 17.68 where the maximum exponent becomes negative and trajectories fall to convergence to the equilibria.

This result can be justified using bifurcation diagram and $0-1$ test. Figure 3 shows that following the periodic state, the system experiences a period doubling cascade leading to chaos right in the interval $a \in[13.94,17.68]$. The window in this region corresponds to the small region in the Lyapunov spectrum whereby the maximum exponents become zero temporarily. Figure 4 shows the result of $0-1$ test 


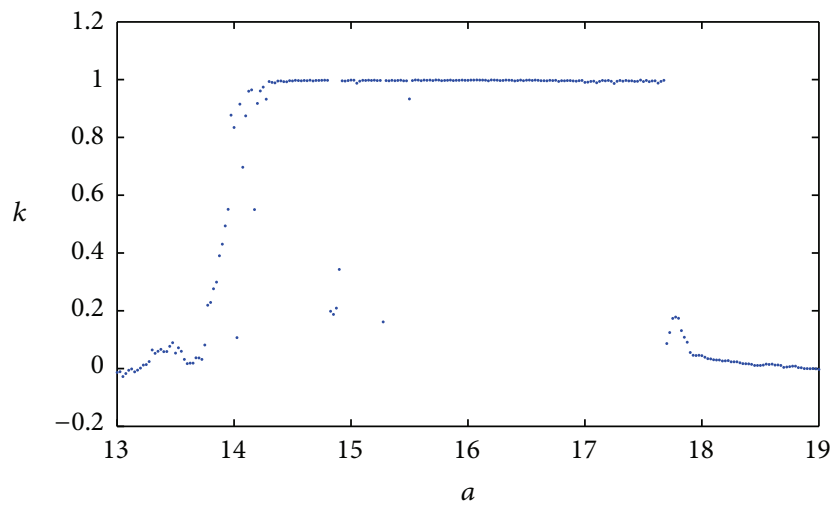

FIGURE 4: Implication of $0-1$ test on the system with respect to $a$.

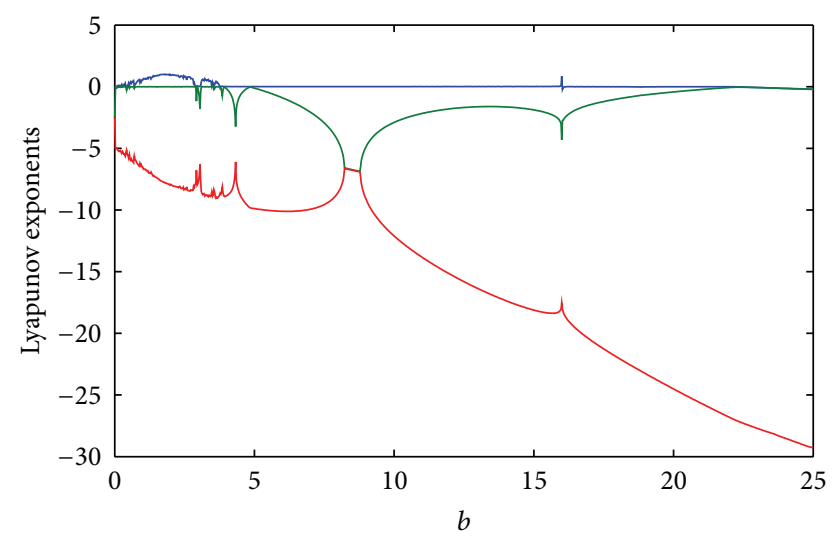

FIGURE 5: Lyapunov exponents spectrum of the system with respect to $b$.

which is clearly in accordance with Figures 2 and 3. Results regarding parameters $b$ and $c$ are displayed in Figures 5, 6, 7, and 8.

\section{Theoretical Proof of Chaos}

4.1. Finding the Heteroclinic Orbit. Numerical simulations do not give us the rigorous proof of the existence of chaos in a system. Therefore, we have to work theoretically to find the final answer to this question. The method we go through here is Shil'nikov theorem for the existence of chaos. Having the conditions of this theorem satisfied, it says that the system has Smale horseshoes and horseshoe type of chaos.

Consider the three-dimensional autonomous system

$$
\dot{x}=f(x),
$$

where vector field $f: \mathbb{R}^{3} \rightarrow \mathbb{R}^{3}$ belongs to class $\mathbb{C}^{r}(r \geq 1)$. Shil'nikov theorem is [13].

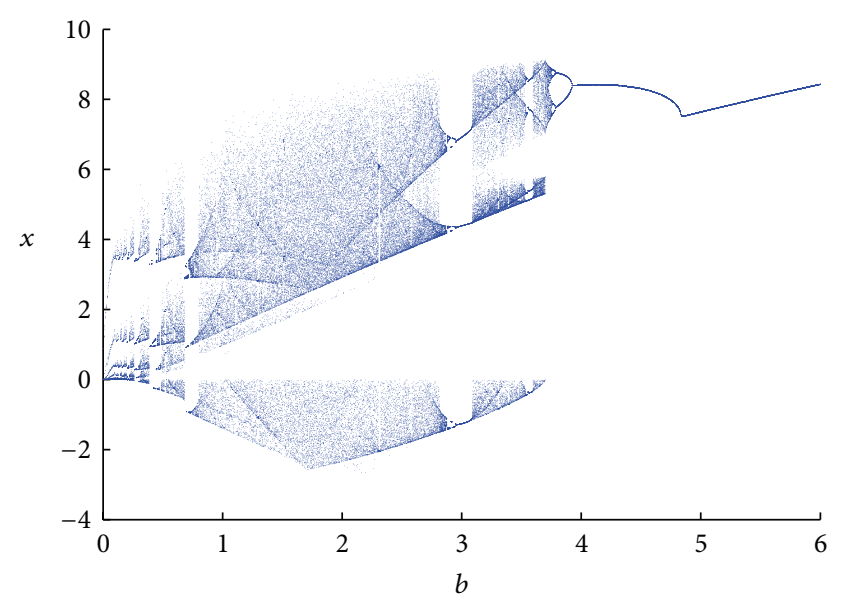

Figure 6: Bifurcation diagram of the system with respect to $b$.

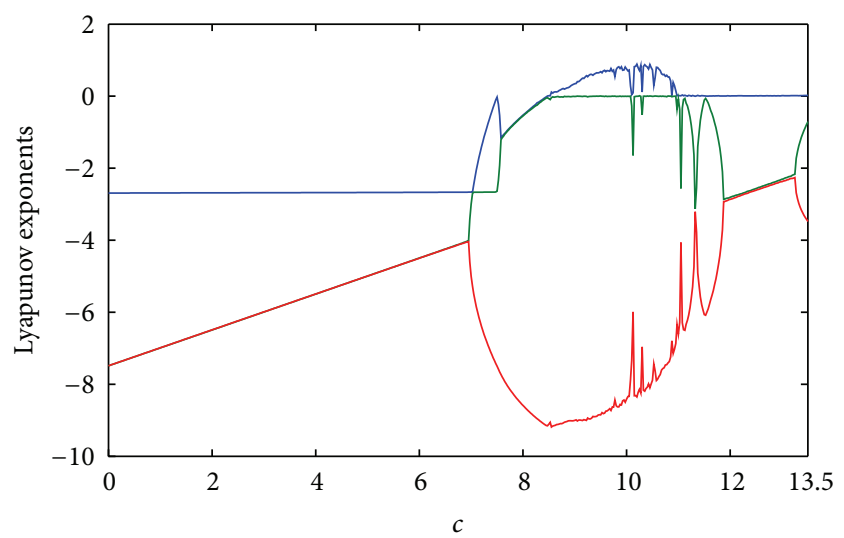

FIGURE 7: Lyapunov exponents spectrum of the system with respect to $c$.

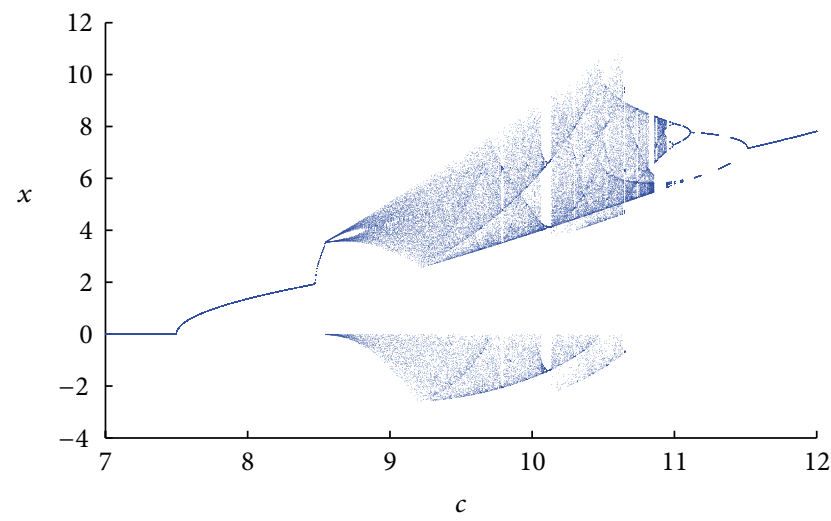

FIGURE 8: Bifurcation diagram of the system with respect to $c$.

Theorem 1 (Heteroclinic Shil'nikov theorem). Suppose that two distinct equilibrium points, denoted by $P_{1}$ and $P_{2}$, respectively, of system $\dot{x}=f(x)$, are saddle foci whose characteristic values $\gamma_{k}, \rho_{k}+i \omega_{k},(k=1,2)$ satisfy the Shil'nikov inequalities 
$\rho_{1} \rho_{2}>0$ or $\gamma_{1} \gamma_{2}>0$. Suppose also that there exists a heteroclinic orbit jointing $P_{1}$ and $P_{2}$. Then the system $\dot{x}=f(x)$ has both Smale horseshoes and the horseshoe type of chaos.

Since the origin is not a saddle focus, Shil'nikov theorem cannot be applied on it. However, $v_{2,3}$ and $v_{4,5}$ satisfy the theorem's condition. Here we put $v_{4,5}$ in our consideration and show the presence of a heteroclinic orbit between them which proves the existence of chaos.

The method we employ to find the heteroclinic orbit is undetermined coefficients method [8] with a new approach [14]. Without loss of generality, assume the direction from $v_{4}$ to $v_{5}$ corresponding to $t \rightarrow \infty$ and from $v_{5}$ to $v_{4}$ corresponding to $t \rightarrow-\infty$.

In the case of $t>0$ and according to the undetermined coefficients method, assume that

$$
\begin{array}{r}
x(t)=x_{5}+\sum_{k=1}^{\infty} \bar{a}_{k} e^{k \alpha t}, \\
y(t)=y_{5}+\sum_{k=1}^{\infty} \bar{b}_{k} e^{k \alpha t}, \\
z(t)=z_{5}+\sum_{k=1}^{\infty} \bar{c}_{k} e^{k \alpha t}, \\
t>0 .
\end{array}
$$

Substituting these equations into the system (4) we have

$$
\begin{aligned}
& \sum_{k=1}^{\infty} \bar{a}_{k} k \alpha e^{k \alpha t} \\
& =a\left(y_{5}+\sum_{k=1}^{\infty} \bar{b}_{k} e^{k \alpha t}-x_{5}-\sum_{k=1}^{\infty} \bar{a}_{k} e^{k \alpha t}\right)+y_{5} z_{5} \\
& +y_{5} \sum_{k=1}^{\infty} \bar{c}_{k} e^{k \alpha t}+z_{5} \sum_{k=1}^{\infty} \bar{b}_{k} e^{k \alpha t}+\sum_{i+j=k \geq 2}^{\infty} \bar{b}_{i} \bar{c}_{j} e^{k \alpha t}, \\
& \sum_{k=1}^{\infty} \bar{b}_{k} k \alpha e^{k \alpha t} \\
& =(c-a) x_{5}+(c-a) \sum_{k=1}^{\infty} \bar{a}_{k} e^{k \alpha t}+c y_{5} \\
& +c \sum_{k=1}^{\infty} \bar{b}_{k} e^{k \alpha t}-x_{5} z_{5}-x_{5} \sum_{k=1}^{\infty} \bar{c}_{k} e^{k \alpha t} \\
& -z_{5} \sum_{k=1}^{\infty} \bar{a}_{k} e^{k \alpha t}-\sum_{i+j=k \geq 2}^{\infty} \bar{a}_{i} \bar{c}_{j} e^{k \alpha t}, \\
& \sum_{k=1}^{\infty} \bar{c}_{k} k \alpha e^{k \alpha t} \\
& =x_{5} y_{5}+x_{5} \sum_{k=1}^{\infty} \bar{b}_{k} e^{k \alpha t}+y_{5} \sum_{k=1}^{\infty} \bar{a}_{k} e^{k \alpha t} \\
& +\sum_{i+j=k \geq 2}^{\infty} \bar{a}_{i} \bar{b}_{j} e^{k \alpha t}-b z_{5}-b \sum_{k=1}^{\infty} \bar{c}_{k} e^{k \alpha t} .
\end{aligned}
$$

Comparing the coefficients of $e^{k \alpha t}$ of the same power we have

$$
\begin{gathered}
\left(\alpha I-D f\left(v_{5}\right)\right) \cdot\left(\begin{array}{l}
\bar{a}_{1} \\
\bar{b}_{1} \\
\bar{c}_{1}
\end{array}\right)=0, \quad \text { for } k=1, \\
\left(k \alpha I-D f\left(v_{5}\right)\right) \cdot\left(\begin{array}{c}
\bar{a}_{k} \\
\bar{b}_{k} \\
\bar{c}_{k}
\end{array}\right)=\left(\begin{array}{c}
\sum_{i+j=k} \bar{b}_{i} \bar{c}_{j} \\
-\sum_{i+j=k} \bar{a}_{i} \bar{c}_{j} \\
\sum_{i+j=k} \bar{a}_{i} \bar{b}_{j}
\end{array}\right)
\end{gathered}
$$

which holds for $k \geq 2$. In (15), $\left(\bar{a}_{1}, \bar{b}_{1}, \bar{c}_{1}\right) \neq 0$ because otherwise, by induction we get

$$
\left(\bar{a}_{k}, \bar{b}_{k}, \bar{c}_{k}\right)=0
$$

for all $k \geq 2$. In addition, since jacobian at $v_{5}$ has a negative eigenvalue, there is a unique $\alpha$ such that

$$
\operatorname{det}\left(\alpha I-D f\left(v_{5}\right)\right)=0 .
$$

Thus, we can easily determine $\bar{a}_{1}, \bar{b}_{1}$, and $\bar{c}_{1}$ using a free variable $\theta$. Also note that in (16)

$$
\operatorname{det}\left(k \alpha I-D f\left(v_{5}\right)\right) \neq 0 \text {. }
$$

Now we consider $t<0$, corresponding to moving from $v_{5}$ to $v_{4}$. Using the variable transformation $\tau=-t$ with $t>0$, system (4) transforms to

$$
\begin{gathered}
\frac{d x}{d \tau}=a(x-y)-y z \\
\frac{d y}{d \tau}=(a-c) x-c y+x z \\
\frac{d z}{d \tau}=-x y+b z
\end{gathered}
$$

Similarly assume that

$$
\begin{array}{r}
x(t)=x_{4}+\sum_{k=1}^{\infty} \widehat{a}_{k} e^{-k \beta \tau}, \\
y(t)=y_{4}+\sum_{k=1}^{\infty} \widehat{b}_{k} e^{-k \beta \tau}, \\
z(t)=z_{4}+\sum_{k=1}^{\infty} \widehat{c}_{k} e^{-k \beta \tau}, \\
\tau<0 .
\end{array}
$$


Substituting these equations into the system (20) we obtain

$$
\begin{aligned}
& \sum_{k=1}^{\infty}-\widehat{a}_{k} k \beta e^{-k \beta \tau} \\
& =a x_{4}+a \sum \widehat{a}_{k} e^{-k \beta \tau}-a y_{4}-a \sum_{k=1}^{\infty} \widehat{b}_{k} e^{-k \beta \tau} \\
& -y_{4} z_{4}-y_{4} \sum_{k=1}^{\infty} \widehat{c}_{k} e^{-k \beta \tau}-z_{4} \sum_{k=1}^{\infty} \widehat{b}_{k} e^{-k \beta \tau} \\
& -\sum_{i+j=k \geq 2}^{\infty} \widehat{c}_{i} \widehat{b}_{j} e^{-k \beta \tau} \\
& \sum_{k=1}^{\infty}-\widehat{b}_{k} k \beta e^{-k \beta \tau} \\
& =(a-c)\left(x_{4}+\sum_{k=1}^{\infty} \widehat{a}_{k} e^{-k \beta \tau}\right) \\
& -c\left(y_{4}-\sum_{k=1}^{\infty} \widehat{b}_{k} e^{-k \beta \tau}\right) \\
& +x_{4}\left(z_{4}+\sum_{k=1}^{\infty} \widehat{c}_{k} e^{-k \beta \tau}\right)+z_{4} \sum_{k=1}^{\infty} \widehat{a}_{k} e^{-k \beta \tau} \\
& +\sum_{i+j=k \geq 2}^{\infty} e^{-k \beta \tau} \\
& \sum_{k=1}^{\infty}-\widehat{c}_{k} k \beta e^{-k \beta \tau} \\
& =-x_{4} y_{4}-x_{4} \sum_{k=1}^{\infty} \widehat{b}_{k} e^{-k \beta \tau}-y_{4} \sum_{k=1}^{\infty} \widehat{a}_{k} e^{-k \beta \tau} \\
& -\sum_{i+j=k \geq 2}^{\infty} \widehat{a}_{i} \widehat{b}_{j} e^{-k \beta \tau}+b z_{4}+b \sum_{k=1}^{\infty} \widehat{c}_{k} e^{-k \beta \tau}
\end{aligned}
$$

Now we equate the coefficients of $e^{-k \beta \tau}$ which have same power terms. It gives us the following:

$$
\begin{gathered}
\left(\beta I-D f\left(v_{4}\right)\right) \cdot\left(\begin{array}{l}
\widehat{a}_{1} \\
\widehat{b}_{1} \\
\widehat{c}_{1}
\end{array}\right)=0, \quad \text { for } k=1, \\
\left(k \beta I-D f\left(v_{4}\right)\right) \cdot\left(\begin{array}{c}
\widehat{a}_{k} \\
\widehat{b}_{k} \\
\widehat{c}_{k}
\end{array}\right)=\left(\begin{array}{c}
-\sum_{i+j=k} \widehat{b}_{i} \widehat{c}_{j} \\
\sum_{i+j=k} \widehat{a}_{i} \widehat{c}_{j} \\
-\sum_{i+j=k} \widehat{a}_{i} \widehat{b}_{j}
\end{array}\right), \quad \text { for } k \geq 2 .
\end{gathered}
$$

Similar to the previous case, $\widehat{a}_{1}, \widehat{b}_{1}$, and $\widehat{c}_{1}$ can be determined by a free variable and $\operatorname{det}\left(k \beta I-D f\left(v_{4}\right)\right) \neq 0$.
So the final expression of the heteroclinic orbit connecting $v_{4}$ and $v_{5}$ is

$$
\begin{aligned}
& x(t)= \begin{cases}x_{5}+\sum_{k=1}^{\infty} \bar{a}_{k}(a, b, c, \alpha) e^{k \alpha t}, & t>0, \\
x_{4}+\sum_{k=1}^{\infty} \widehat{a}_{k}(a, b, c, \beta) e^{-k \beta t}, & t<0,\end{cases} \\
& y(t)= \begin{cases}y_{5}+\sum_{k=1}^{\infty} \bar{b}_{k}(a, b, c, \alpha) e^{k \alpha t}, & t>0, \\
y_{4}+\sum_{k=1}^{\infty} \widehat{b}_{k}(a, b, c, \beta) e^{-k \beta t}, & t<0,\end{cases} \\
& z(t)= \begin{cases}z_{5}+\sum_{k=1}^{\infty} \bar{c}_{k}(a, b, c, \alpha) e^{k \alpha t}, & t>0, \\
z_{4}+\sum_{k=1}^{\infty} \widehat{c}_{k}(a, b, c, \beta) e^{-k \beta t}, & t<0 .\end{cases}
\end{aligned}
$$

4.2. Convergence of the Series Solution. Since the solution is in series form, we have to prove that it is convergence before making any conclusion. Having (15) and (16) and the fact that $\left(k \alpha I-D f\left(v_{5}\right)\right)$ is nonsingular, for any $k \geq 1$ we have

$$
\left(\begin{array}{l}
\bar{a}_{k} \\
\bar{b}_{k} \\
\bar{c}_{k}
\end{array}\right)=\left(\begin{array}{l}
m_{a} \theta^{k} \\
m_{b} \theta^{k} \\
m_{c} \theta^{k}
\end{array}\right),
$$

where $m_{a}, m_{b}$, and $m_{c}$ are constants and $\theta$ is the free variable we use to determine $a_{1}, b_{1}$, and $c_{1}$. In addition, after doing some algebra on (16) using the default values of $a, b$, and $c$ we have

$$
\bar{a}_{k}<\frac{M \theta^{k}}{k}, \quad k \geq 2 .
$$

As we mentioned before, $\theta$ is a free variable, so we can assume it to be $0<\theta<1$. Thus

$$
\begin{aligned}
x(t)= & x_{5}+\sum_{k=1}^{\infty} \bar{a}_{k} e^{k \alpha t} \\
< & x_{5}+\bar{a}_{1} e^{\alpha t}+\sum_{k=2}^{\infty} \frac{M \theta^{k}}{k} e^{k \alpha t} \\
< & x_{5}+\bar{a}_{1} e^{\alpha t}+M \theta \sum_{k=2}^{\infty} \frac{e^{k \alpha t}}{k} \\
= & x_{5}+\bar{a}_{1} e^{\alpha t} \\
& \quad+M \theta\left(-\ln \left(1-e^{\alpha t}-e^{\alpha t}\right)\right)<x_{5}+\bar{a}_{1}+M \theta .
\end{aligned}
$$

Exactly the same result holds for the second part of $x(t)$ corresponding to $t<0$. Therefore the convergence of the series solution of $x(t)$ is proved. Similar procedure can be done to prove the convergence of $y(t)$ and $z(t)$. 
One remark is necessary to give here. If we consider $v_{2}$ and $v_{3}$, we see that they satisfy in Shil'nikov theorem condition and we can follow just the same algebraic procedure to find the heteroclinic orbit between them. However, the location of these two equilibrium points are very far from the chaotic attractor observed. Therefore more investigation is required to find out the role of this heteroclinic orbit on the dynamic of the chaotic system (4).

\section{Conclusion}

In this paper, a new 3D quadratic chaotic attractor was introduced and analysed. We studied basic properties of this system by means of Jacobian matrices, Lyapunov exponents, and various definitions of fractal dimension which showed topological distinction between this attractor and some other well-known systems. In addition, more detailed information on its dynamic was obtained using Lyapunov exponents spectrum, bifurcation diagrams, and 0-1 chaos indicator test that disclosed just a portion of the underlying dynamic of this system. And finally, we proved the existence of chaos by showing that this system has Smale horseshoes and horseshoe type of chaos.

\section{References}

[1] E. Lorenz, "Deterministic nonperiodic flow," Journal of the Atmospheric Sciences, vol. 20, no. 2, pp. 130-141, 1963.

[2] C. Sparrow, The Lorenz Equations: Bifurcations, Chaos, and Strange Attractors, vol. 41 of Applied Mathematical Sciences, Springer, New York, NY, USA, 1982.

[3] Z. Wei, "Dynamical behaviors of a chaotic system with no equilibria," Physics Letters A, vol. 376, no. 2, pp. 102-108, 2011.

[4] X. Wang and G. Chen, "A chaotic system with only one stable equilibrium," Communications in Nonlinear Science and Numerical Simulation, vol. 17, no. 3, pp. 1264-1272, 2012.

[5] X. Wang and G. Chen, "Constructing a chaotic system with any number of equilibria," Nonlinear Dynamics, vol. 71, pp. 429-436, 2013.

[6] J. Lü and G. Chen, "A new chaotic attractor coined," International Journal of Bifurcation and Chaos, vol. 12, no. 3, pp. 659661, 2002.

[7] G. Qi, G. Chen, S. Du, Z. Chen, and Z. Yuan, "Analysis of a new chaotic system," Physica A, vol. 352, no. 2-4, pp. 295-308, 2005.

[8] T. Zhou, Y. Tang, and G. Chen, "Chen's attractor exists," International Journal of Bifurcation and Chaos, vol. 14, no. 9, pp. 3167-3177, 2004.

[9] V. Govorukhin, "Calculation Lyapunov exponents for ODE," 2004, http://www.mathworks.com/matlabcentral/fileexchange/ 4628.

[10] A. Wolf, J. B. Swift, H. L. Swinney, and J. A. Vastano, "Determining Lyapunov exponents from a time series," Physica D, vol. 16, no. 3, pp. 285-317, 1985.

[11] J. Kaplan and J. A. Yorke, "Chaotic behavior of multidimensional difference equations," in Functional Differential Equations and Approximation of Fixed Points, vol. 730 of Lecture Notes in Mathematics, pp. 204-227, Springer, Berlin, Germany, 1979.

[12] G. A. Gottwald and I. Melbourne, "On the implementation of the 0-1 test for chaos," SIAM Journal on Applied Dynamical Systems, vol. 8, no. 1, pp. 129-145, 2009.
[13] E. Zeraoulia and J. C. Sprott, 2-D Quadratic Maps and 3-D ODE Systems, vol. 73, World Scientific Publishing, Hackensack, NJ, USA, 2010.

[14] X. Wang, J. Li, and J. Fang, "Shil'nikov Chaos of a 3-D quadratic autonomous system with a four-wing chaotic attractor," in Proceedings of the 30th Chinese Control Conference (CCC '11), pp. 561-565, 2011. 


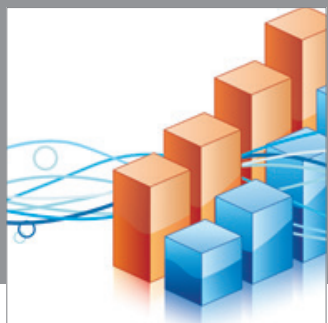

Advances in

Operations Research

mansans

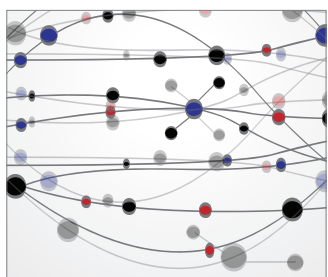

The Scientific World Journal
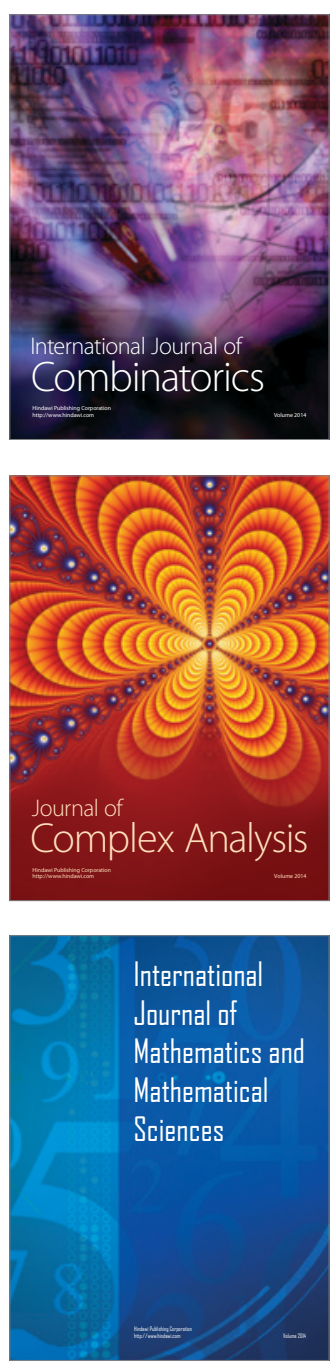
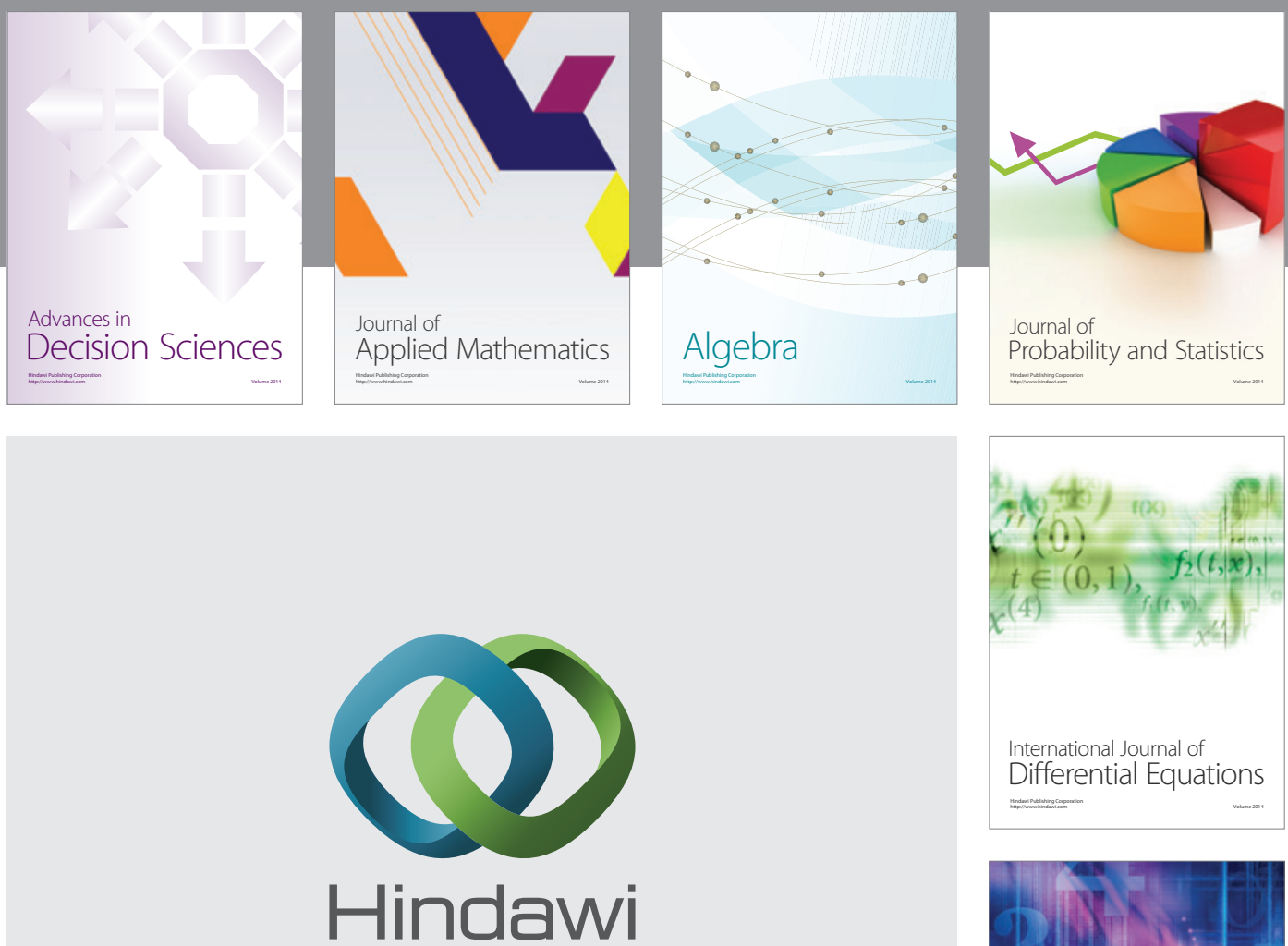

Submit your manuscripts at http://www.hindawi.com
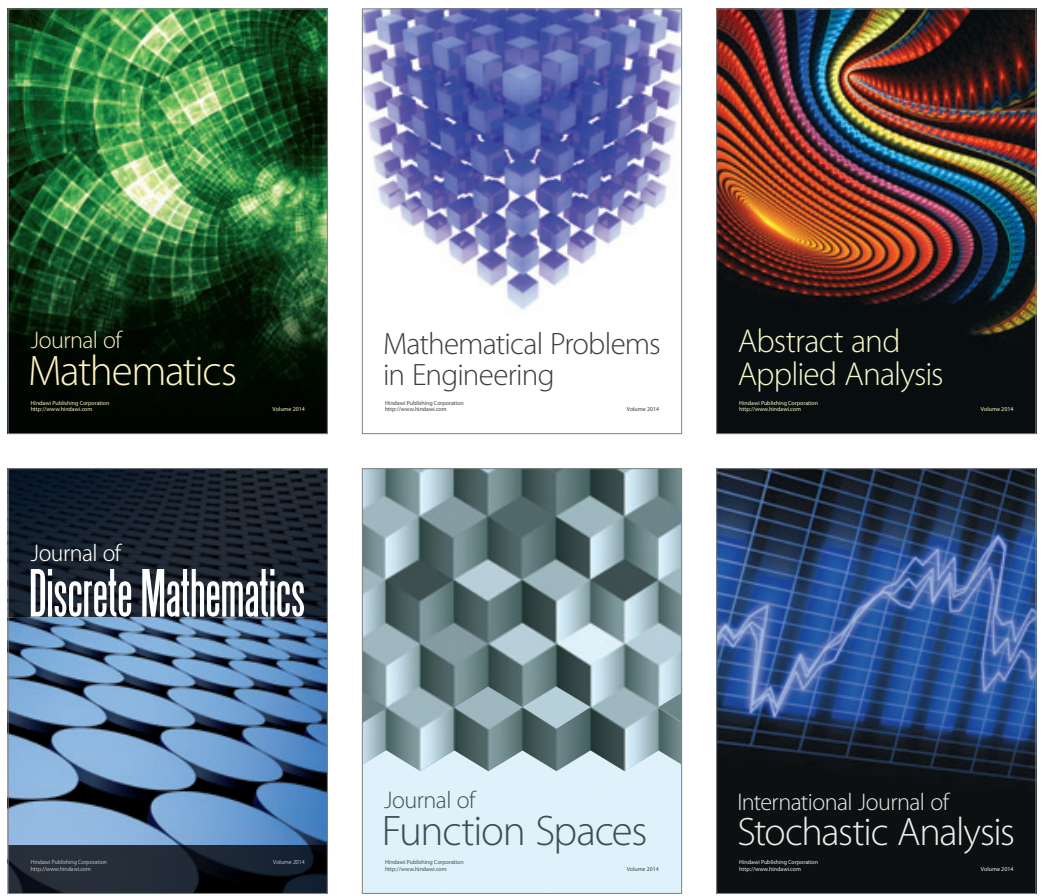

Journal of

Function Spaces

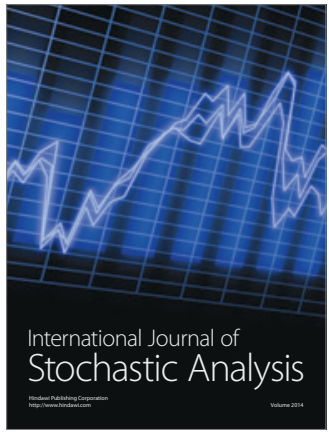

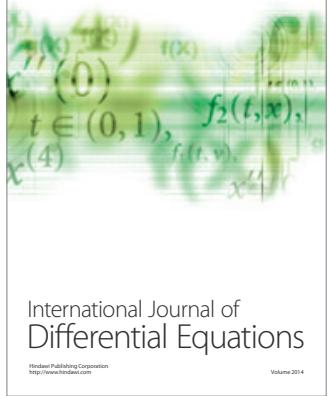
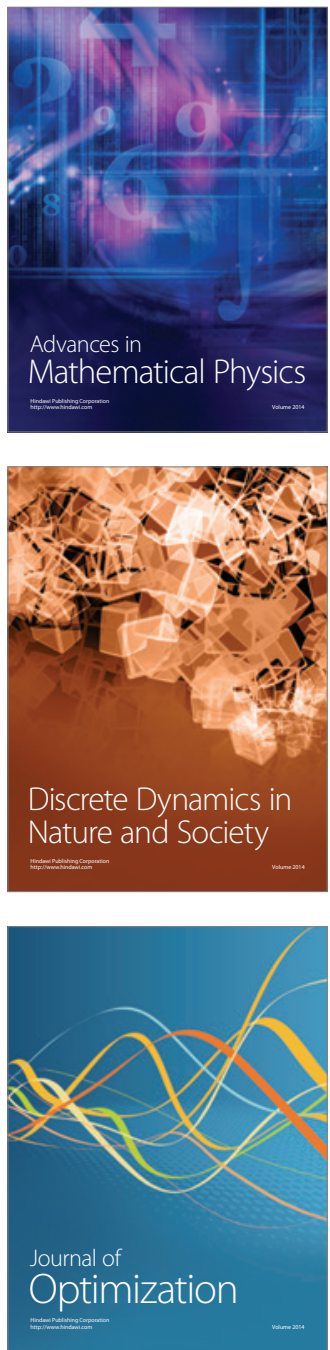